\title{
Synthesizing East African land-cover change over the past 6000 years
}

\author{
Rob Marchant ${ }^{1}$ and Stephen Rucina ${ }^{2}$ \\ East African Land Cover6K, Nairobi, Kenya, 22-23 October 2015
}

The East Africa LandCover6k workshop brought together over 60 experts from the fields of archeology, archeobotany, linguistics and paleoenvironmental studies to synthesize information on East African land-cover change over the past 6000 years. Through an exchange of ideas, the interdisciplinary group aims to understand directions and drivers of land-cover change, and the interaction with various cultures, technologies, and subsistence strategies across the East African region. The workshop outputs were a series of templates for capturing information on land-use and socioecological change that are currently being populated by the wider community. Ultimately this will lead to a series of land-cover maps for Eastern Africa collated within a review manuscript and harmonized with parallel initiatives from other parts of the African continent. The participants also discussed the utility of East African landcover-change data for a range of different disciplines, particularly for stakeholders outside of academia concerned with contemporary issues such as resource use and conservation.

The workshop focused on the following questions: (1) What were the dominant landcover changes during the past 6000 years? (2) What are the key gaps in the archeological and paleoenvironmental records? (3) Were the drivers of land-cover changes natural or anthropogenic? (4) What contemporary questions about human-environment interaction can our data and modeling initiatives help to address?

As one of the core aims of the LandCover6k project is to provide global maps of land cover to feed into climate-model development, Jed Kaplan (U. Lausanne, Switzerland) provided a model-based perspective on land-cover changes in West Africa over the past 6000 years. We then had two overview presentations on the current status and future directions of archeological and paleoecological research in Eastern Africa by Paul Lane (Uppsala U., Sweden) and Dan Olago (U. Nairobi, Kenya) respectively. The workshop then moved to a series of country-specific sessions where current overviews on archeology and paleoecology data were presented as a series of paired talks for Central Africa, Tanzania, Uganda and Kenya.

The second day of the workshop focused on the wider application of land-use data for conservation by Cassian Mumbi (Tanzania Wildlife Research Institute) and Jaqueline Barnard (Kenya Wildlife Service). We then focused on the link to natural resource use specifically on water by Dickens Odeny (National Water Towers Project, Kenya), Philip Omondi (Kenya Meteorological Department), and carbon by Jennifer Farmer (ALTER Carbon project, Uganda).

Workshop participants then divided into three groups. The first, led by Jed Kaplan, focused on developing a matrix to capture data (stratigraphic, radiocarbon, proxy, artefacts and land use) and meta information (site location, age range covered and environmental setting) that has been subsequently developed and will underpin the synthesis. Rob Marchant led another group on collating information around phases of rapid environmental or technological and land-cover change, particularly looking at the periods around 4000 and 800 years BP. Stephen Rucina led the final group, looking at the spread of archeological data and how this can be used for land-use change study.

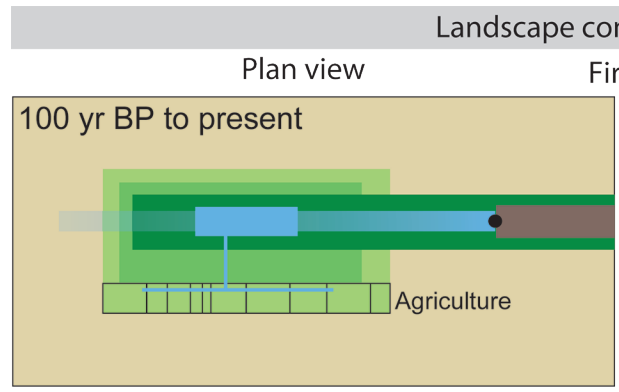

The East Africa LandCover6k initiative is currently in the phase of collating data and information behind land use that will be discussed at forthcoming congresses and feed into the wider development of the LandCover6k project.

For more information on the workshop, or to be involved in the East Africa LandCover6k project, please contact Rob Marchant: Robert.marchant@york.ac.uk

\section{AFFILIATIONS}

'Environment Department, University of York, Heslington, UK

${ }^{2}$ National Museums of Kenya, Nairobi, Kenya

CONTACT

Rob Marchant: robert.marchant@york.ac.uk

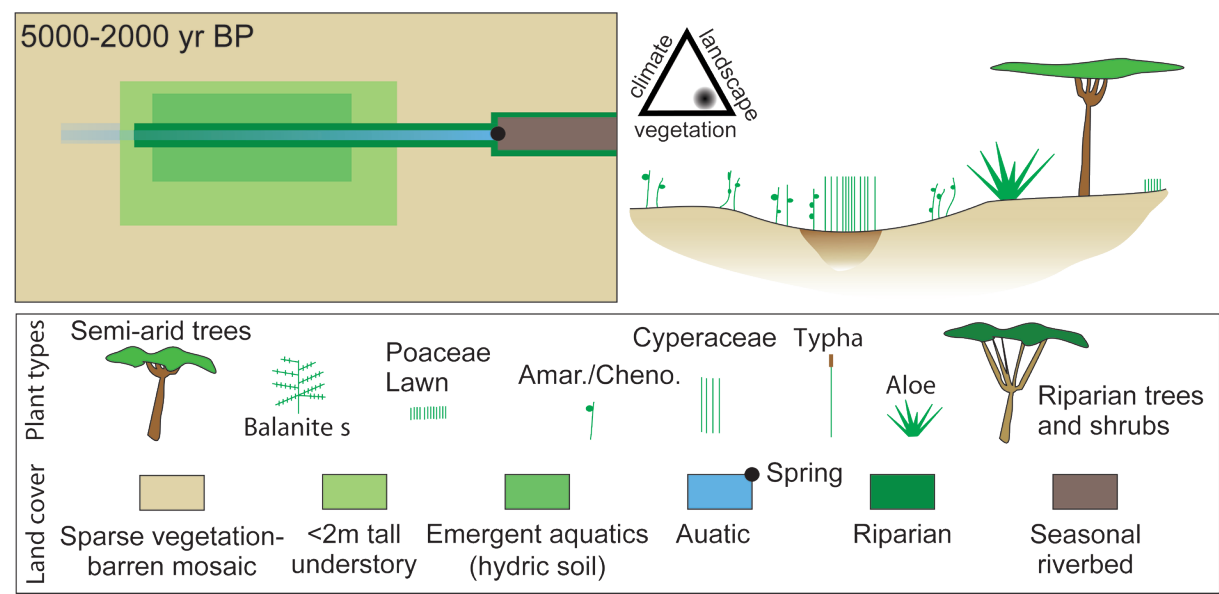

Figure 1: Landscape change inferred from a dated pollen record taken at Esambu swamp, Amboseli, southern Kenya. The mid to late Holocene landscape was characterized by open semi-arid woodland with disjointed fuel connectivity. Through the late Holocene, the catchment was increasingly modified by human activity characterized by channeling water resources and expanding agriculture with fire activity increased. 